\title{
Research on Frequency Measurement of Power System Based on Support Vector Machine
}

\author{
Rui-peng Yang \\ Department of Electrical Engineering,North China Electric Power University,Baoding,China \\ 524347954@qq.com
}

Keywords: Support vector machine; Frequency measurement; Power system; Fitting; FFT

Abstract. A new method for measuring the frequency of power system based on support vector machine is proposed in this paper. This method used the exploratory way, under rare sampling points, to fit the original signal with a single frequency curve. Then, through comparing the amplitude, the power system frequency can be accurately detected, which is simple and of high accuracy.

\section{Introduction}

Power system frequency generates an important impact on operation, control and protection of the power system, and it is the important index of weighing power quality, so it is very necessary to measure it accurately. The each method used now has advantage or disadvantage in the speed and precision of measurement, computation load, the ability to restrain harmonic and the difficult or easy degree to achieve, and it is difficult to give attention to all[1]. Thus, this paper introduced the support vector method (SVM) to measure the frequency of power system, which is simple and of high accuracy.

\section{Support vector machine}

Support vector method is the updated and more effective part of statistical learning theory, and the core of it was put forward from 1992 to 1995 and is still in constant developing stage at present. The theory based on the principle of structure risk minimization provides a new perspective in machine learning, and has been widely applied to pattern recognition and function fitting. The basic idea of SVM for recognition is to map the input space into a high dimensional feature space via nonlinear mapping and to do linear regression in this space for the purpose of approaching primitive function. This algorithm is a convex quadratic optimization problem and can ensure that the obtained solution is the global optimal solution[2].

Model construction.

For support vector machines function regression, firstly, it needs considering the problem of using linear regression function $f(x)=w x+b$ to fitting the data $\left\{x_{i}, y_{i}\right\}, i=1,2, \cdots, n, x_{i} \in R^{d}$, $y_{i} \in R$.

$$
\begin{aligned}
& \text { Set } C=\left\lfloor C_{1}, C_{2}, \cdots, C_{N_{w}}\right\rfloor, \\
& D=\left\lfloor D_{1}, D_{2}, \cdots, D_{N_{w}}\right\rfloor, \\
& w=[C, D],
\end{aligned}
$$




$$
x_{i}=\left\lfloor\cos w_{1} t_{i}, \cdots, \cos w_{N_{w}} t_{i}, \sin w_{1} t_{i}, \cdots, \sin w_{N_{w}} t_{i}\right\rfloor .
$$

And this problem is transformed into solving the frequency decomposition model of discrete time series $\left\{x_{i}, y_{i}\right\}, i=1,2, \cdots, N$.

$$
y_{i}=\sum_{m=1}^{N_{\mathrm{w}}} A_{m} \cos \left(w_{m} t_{i}-\varphi_{m}\right)+e_{i}=\sum_{m=1}^{N_{\mathrm{w}}}\left(C_{m} \cos w_{m} t_{i}+D_{m} \sin w_{m} t_{i}\right)+e_{i}
$$

Where $A_{m}$ and $\varphi_{m}$ are respectively the amplitude and photo corner of the component of the angular frequency $w_{m} ; e_{i}$ represents the error of $t_{i}$ time model, $e_{i}=y_{i}-w x_{i}^{T}, C_{m}=A_{m} \cos \varphi_{m}$, $D_{m}=A_{m} \sin \varphi_{m}$. Accordingly, when the signal contains the component whose angular frequency is $w_{m}$, the value of $A_{m}$ is not equals to 0 . The objective function of this model can be expressed as:

$$
\min \frac{1}{2} \sum_{m=1}^{N_{\mathrm{w}}}\left(C_{m}^{2}+D_{m}^{2}\right)+\frac{c}{2} \sum_{i=1}^{N}\left(\xi_{i}^{(k)}+\xi_{i}^{*(k)}\right)
$$

The constraint is shown as follows:

$$
\left\{\begin{array}{l}
y_{i}-\sum_{m=1}^{N_{\mathrm{w}}}\left(C_{m} \cos w_{m} t_{i}+D_{m} \sin w_{m} t_{i}\right) \leq \varepsilon+\xi_{i} \\
\sum_{m=1}^{N_{\mathrm{w}}}\left(C_{m} \cos w_{m} t_{i}+D_{m} \sin w_{m} t_{i}\right)-y_{i} \leq \varepsilon+\xi_{i}^{*} \\
\xi_{i}, \xi_{i}^{*} \geq 0
\end{array}\right.
$$

The first term in Eq. (2) is make the function more flat and improve the generalization ability. The second term is the loss function where $c$ represents the fault-tolerant penalty coefficient, $\xi_{i}$ and $\xi_{i}^{*}$ represent the slack variable, $\xi^{(k)}$ represents the $k$ times loss function. In the SVM regression, the loss functions commonly used include linear $\varepsilon$ insensitive loss function, second $\varepsilon$ insensitive loss function and Huber loss function. In actual usage, the main choice of the appropriate loss function is according to the characteristics of the actual model. This paper selected the second $\varepsilon$ insensitive loss function:

$$
\begin{aligned}
& \xi^{(2)}=\left\{\begin{array}{l}
0, \quad y-f(x) \leq \varepsilon \\
|y-f(x)|^{2}-\varepsilon, y-f(x) \geq \varepsilon
\end{array}\right. \\
& \xi^{*(2)}=\left\{\begin{array}{l}
0, y-f(x) \geq-\varepsilon \\
|y-f(x)|^{2}-\varepsilon, y-f(x) \leq-\varepsilon
\end{array}\right.
\end{aligned}
$$

\section{Parameter solution.}

If the value of $\xi_{i}^{(*)}$ is low than 0 , set $\xi_{i}^{(*)}=0$. The first two constraints in Eq. (3) still remain, 
and this change will reduce the value of the objective function. Thus, the Langrange function is introduced to solve the optimization problem of Eq. (2) and Eq. (3), which is expressed as follows:

$$
L=\frac{1}{2}\|w\|^{2}+\frac{c}{2} \sum_{i=1}^{N}\left(\xi_{i}^{2}+\xi_{i}^{*(2)}\right)+\sum_{i=1}^{N} \alpha_{i}\left(y_{i}-w x_{i}^{T}-\varepsilon-\xi_{i}\right)+\sum_{i=1}^{N} \alpha_{i}^{*}\left(w x_{i}^{T}-y_{i}-\varepsilon-\xi_{i}^{*}\right)
$$

Where $\alpha_{i}$ and $\alpha_{i}^{*}$ are the Langrange constant. According to $\left(\partial L / \partial \xi_{i}^{(*)}\right)=0$, it has $c\left(\xi_{i}^{(*)}+\varepsilon\right)-\alpha_{i}^{(*)}=0$. Then, set $\lambda_{i}=2 \alpha_{i} /\left(e_{i}-\varepsilon\right)$ and $\lambda_{i}^{*}=2 \alpha_{i}^{*} /\left(-e_{i}-\varepsilon\right)$, there are:

$$
\begin{aligned}
& \lambda_{i}=\left\{\begin{array}{l}
0, e_{i} \leq \varepsilon \\
2 c e_{i} /\left(e_{i}-\varepsilon\right), \text { else }
\end{array}, \lambda_{i}^{*}=\left\{\begin{array}{l}
0, e_{i} \geq-\varepsilon \\
-2 c e_{i} /-\left(e_{i}-\varepsilon\right), \text { else }
\end{array}\right.\right. \\
& L=\frac{1}{2}\|w\|^{2}+\frac{1}{2} \sum_{i=1}^{N}\left(\lambda_{i}\left(e_{i}-\varepsilon\right)^{2}+\lambda_{i}^{*}\left(-e_{i}-\varepsilon\right)^{2}\right)+\frac{c}{2} \sum_{i=1}^{N}\left(\xi_{i}^{2}+\xi_{i}^{* 2}\right)-\sum_{i=1}^{N}\left(\alpha_{i} \xi_{i}+\alpha_{i}^{*} \xi_{i}^{*}\right)
\end{aligned}
$$

Because the value of $\lambda_{i}^{(*)}$ and $\alpha_{i}^{(*)}$ are constant in each iteration, there is:

$$
\frac{\partial L}{\partial w}=w-\sum_{i=1}^{N} x_{i}^{T}\left(\lambda_{i}+\lambda_{i}^{*}\right) e_{i}+\sum_{i=1}^{N} x_{i}^{T}\left(\lambda_{i}-\lambda_{i}^{*}\right) \varepsilon=w+X D_{\left(\lambda_{i}+\lambda_{i}^{*}\right)} w X^{T}-x D_{\left(\lambda_{i}+\lambda_{i}^{*}\right)} y+X D_{\left(\lambda_{i}+\lambda_{i}^{*}\right)} \mathcal{E}
$$

Where $X=\left[x_{1}^{T}, x_{2}^{T}, \cdots x_{i}^{T}\right]$,

$$
D\left(\lambda_{i}+\lambda_{i}^{*}\right)=\left[\begin{array}{cccc}
\lambda_{1}+\lambda_{1}^{*} & 0 & \cdots & 0 \\
0 & \lambda_{2}+\lambda_{2}^{*} & 0 & \vdots \\
\vdots & 0 & \ddots & 0 \\
0 & \cdots & 0 & \lambda_{i}+\lambda_{i}^{*}
\end{array}\right]
$$

$D\left(\lambda_{i}+\lambda_{i}^{*}\right)$ is similar to Eq. (3), we can obtain the following according to $\partial L / \partial w=0$ :

$$
w=\left[I+X D X^{T}\right]^{-1} X\left[D_{\left(\lambda_{i}+\lambda_{i}^{*}\right)} y-D_{\left(\lambda_{i}+\lambda_{i}^{*}\right)} \varepsilon\right]
$$

\section{Algorithm steps.}

From the above, the steps of solving the IRWLS methods can be summed as follows: (1) Set the sampling frequency, the point number of sampling, the initial value of the random sequence $w$, and set the number of iterations $r=0$; (2) Calculate $e_{i}, \alpha_{i}$ and $\alpha_{i}^{*}$; (3) Compute $\lambda_{i}$ and $\lambda_{i}^{*}$; (4) Compute $w_{r+1}$ and set $r=r+1$; (5) Judge the value of $\left\|w_{r+1}-w_{r}\right\|$. If the value is lower than 0.1 , stop operation, otherwise jump to step (2).

\section{Algorithm application}

As can be seen from Eq. (1), the value of $w_{m}$ and $N_{w}$ can be arbitrarily set. Because the power system frequency range is determined, the tentative way is used in this range, and set $N_{w}=1$, use 
different value of $w_{m}$ to find the frequency of power system[3]. The following two examples are used to illustrate that the proposed algorithm can accurately measure the power system frequency under the condition that the sampling points are less, and compared with the FFT.

The case that the signal does not contain harmonics.

Set the signal as $x=\sin (51 \times 2 \pi t-\pi / 6)$, the sampling frequency is $300 \mathrm{~Hz}$, the sampling points are 6 . Set $N_{w}=1$, use one of the value in $45-55 \mathrm{~Hz}$ to be the $f$. The simulation results are shown in Table. 1.

Table. 1 The simulation results of SVM for Case 1

\begin{tabular}{cccccc}
\hline $\mathrm{f} / \mathrm{Hz}$ & 46 & 47 & 48 & 49 & 50 \\
Amplitude/V & 0.54019 & 0.54369 & 0.64579 & 0.53714 & 0.54780 \\
\hline $\mathrm{f} / \mathrm{Hz}$ & 51 & 52 & 53 & 54 & 55 \\
Amplitude/V & 0.54781 & 0.54720 & 0.54600 & 0.54424 & 0.54194 \\
\hline
\end{tabular}

It can be seen that, the different value of $w_{m}$ determine the different amplitude. When the value of $w_{m}$ is 51 , the amplitude is maximum, which indicates that, the curve is fitted the best. Thus, the frequency is $51 \mathrm{~Hz}$.

Under the same circumstances, apply FFT, the simulation results are shown in Fig. 1. It can be seen that FFT algorithm in this condition can not be obtained an accurate frequency. In order to obtain the frequency component of $51 \mathrm{~Hz}$, the frequency of FFT must must reach to $1 \mathrm{~Hz}$, theoretically requires sampling time of at least 1 s (50 power frequency cycles). So the sampling frequency is $300 \mathrm{~Hz}$, the sampling points should be increased to at least 300 .

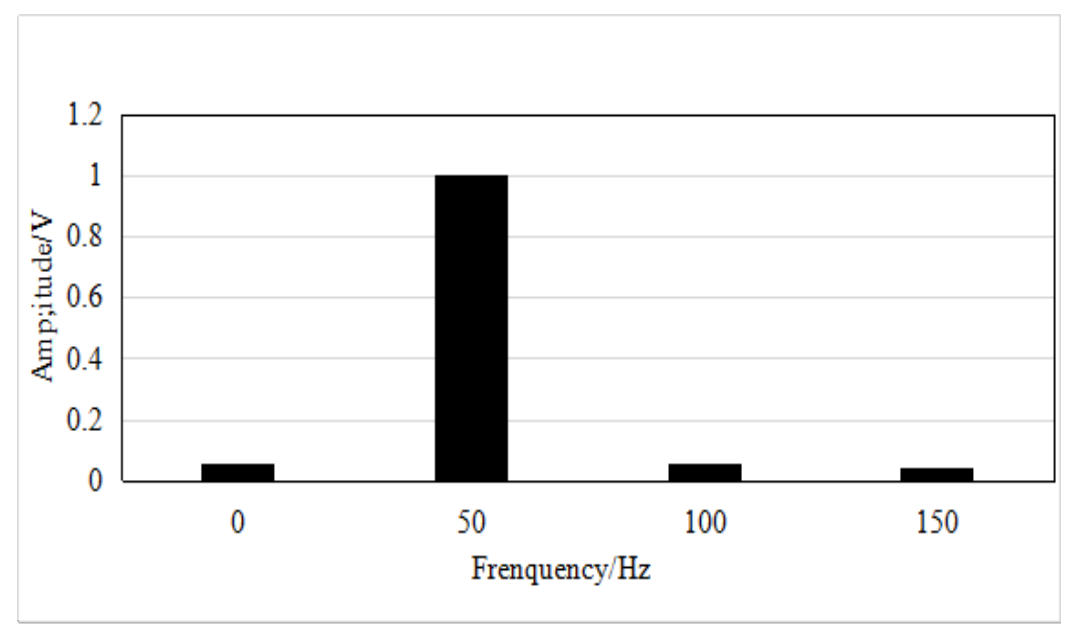

Fig. 1 The simulation results of FFT for Case 1

\section{The case that the signal contains harmonics.}

The actual measuring power system frequency, usually with a low-pass filter will attenuate the higher harmonics to negligible proportions, so the impact is mainly measured 2,3 harmonics, the following signal containing 2, 3 harmonic the case is discussed.

Set the signal as $x=\sin (2 \pi f t-\pi / 6)+0.2 \sin (4 \pi f t-\pi / 7)+0.1 \sin (6 \pi f t-0.1 \pi)$, the sampling frequency is $300 \mathrm{~Hz}$, the sampling points are 14 . Set $N_{w}=1$, use one of the value in $45-55 \mathrm{~Hz}$ to be 
the $f$. The simulation results are shown in Table. 2, which indicate that when the signal contains 2 and 3 harmonic, the proposed method can also accurately detected it out. The simulation results of FFT is shown in Fig. 2.

Table. 2 The simulation results of SVM for Case 2

\begin{tabular}{cccccc}
\hline $\mathrm{f} / \mathrm{Hz}$ & 46 & 47 & 48 & 49 & 50 \\
Amplitude/V & 0.65625 & 0.68910 & 0.61472 & 0.73217 & 0.74115 \\
\hline $\mathrm{f} / \mathrm{Hz}$ & 51 & 52 & 53 & 54 & 55 \\
Amplitude/V & 0.74200 & 0.73554 & 0.72292 & 0.70539 & 0.68412 \\
\hline
\end{tabular}

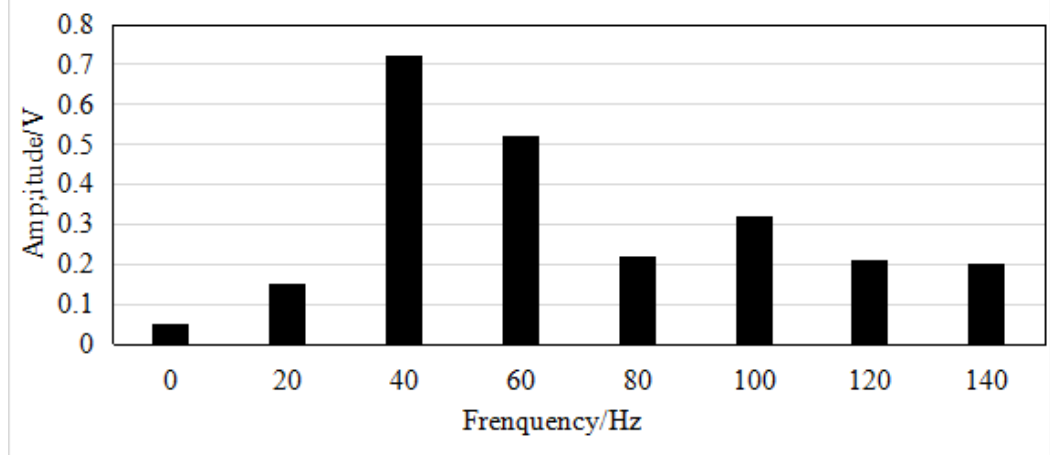

Fig. 2 The simulation results of FFT for Case 2

\section{Conclusions}

This paper uses the linear regression function to fit the signal including harmonic needed to be measured and the iterative reweighted least squares procedure is educed for signal spectrum analysis. Then, a new method to measure power system frequency is presented. In case of the few sample number, this method, using single frequency to sound out whether it can fit the original signal curve well or not, can measure power system frequency with accuracy by comparing different amplitude. Through discussing three examples and comparing with FFT, it can be proved that the new method has the characteristics of simple computation and high precision.

\section{References}

[1] Xie Xiaorong, Han Yingyi. An overview on power system frequency measurement Automation of Electric Power Systems, Volume 23, Issue 3 (1999), P. 54-58.

[2] Zhou Li, Liu Kaipei, Ma Bingwei. New Method to Measure Power System Frequency Based on SVM. High Voltage Engineering, Volume 32, Issue 6 (2006), P. 94-96.

[3] Jin Huafeng, He Benteng. Real TME estimation of power system frequency. Power System Protection and Control, Volume 26, Issue 3 (1998), P. 17-20. 